\title{
PASSIVE ATMOSPHERIC DIFFUSION WITH GAUSSIAN FRAGMENTATION
}

\author{
S.S. Beauchemin, ${ }^{*}$ H.O. Hamshari, ${ }^{*}$ and M.A. Bauer*
}

\begin{abstract}
Events such as atmospheric gas dispersion by industrial accidents or processes are generally predicted with Gaussian plumes, coupled with local models of gas emission. In this contribution we investigate the association of integral models of instantaneous emission with Gaussian dispersion processes for predicting the progression of potentially hazardous low-altitude emissions over sensitive or populated areas. We develop a novel, more accurate approach suitable for dynamic, high spatial resolution atmospheric conditions by means of gas plume fragmentation and parallel estimation of extent and concentration.
\end{abstract}

\section{Key Words}

Parallel simulation, integral model, atmospheric plume, Gaussian cloud, Hexagonal sphere packing

\section{Introduction}

Because of its simplicity, the Gaussian dispersion model is often used for predicting the progression of atmospheric gas plumes [1-3]. This model relies on a number of hypotheses to determine the path and spread of plumes, the most fundamental stating that the dispersion must be passive, which is equivalent to considering the gas density as roughly the same as that of the surrounding atmosphere.

Before reaching the stage of passive dispersion, initial conditions of gas emissions are often addressed differently, as various gases may have densities differing from ambient air (depending on molecular weight, temperature, altitude of emission, and so on). Failing to consider such parameters in the early stages could result in considerable prediction errors, either in concentration levels or geographical spread.

The use of Gaussian dispersion models requires that terrain be free of significant obstacles such as skyscrapers or mountain ranges, or that the altitude of the emission source be sufficiently high to ignore obstacles. Other hypotheses include the absence of atmospheric turbulence and gas densities which minimize the effect of gravity on the plume. Under such conditions, the dispersion results

\footnotetext{
* Department of Computer Science, The University of Western Ontario, 1151 Richmond Street, London ON N6A 5B7; e-mail: beau@csd.uwo.ca, hohamsha@uwo.ca, bauer@csd.uwo.ca Recommended by Prof. Shaharuddin Salleh

(paper no. 202-2338)
}

are usually considered correct from approximately $100 \mathrm{~m}$ from the emitting source and beyond [4].

Hence, in our low-altitude emission framework, the sole use of a Gaussian dispersion model is clearly inadequate. A local emission model for the source is required, and we adopt the integral model as an instantaneous emission source, which aptly provides the initial conditions for the Gaussian dispersion simulation. An additional hypothesis pertaining to the integral model requires that gas emission be relatively significant, generally in the order of a few $\mathrm{m}^{3} / \mathrm{s}$. A gas cloud following an integral model evolves at very small spatio-temporal scales while compared with the much larger dispersion extents inherent to a Gaussian model. However, the integral model is required, if only to provide the dispersion process with adequately realistic initial parameters.

The integral model employs a stack of cylinders, describing volumes (or puffs) containing gas particles. These puffs are updated through an iterative process until they reach the density of the surrounding air. Ultimately, puffs with such densities are injected in the stack of the Gaussian model, for large-scale dispersion computations to begin.

To combine the integral and Gaussian models while preserving a relative flexibility, we choose an approach in which both models share common environmental parameters, operating over a discretized grid map describing dynamic atmospheric and terrain conditions. At each iteration, the combined model updates the environmental conditions and the characteristics of emission sources, and injects gas puffs in the integral or Gaussian model. Results are expressed as concentration and dispersion grids over the region of interest.

This contribution presents a combined integralGaussian model which includes Gaussian puff fragmentation. The process of fragmentation consists of breaking up large Gaussian puffs into sets of smaller ones, to increase the accuracy of plume simulations: Gaussians puffs covering large geographical extents ( $1 \mathrm{~km}$ or more) are generally subjected to spatially variable winds within their extent, while puffs with smaller extents are less likely to suffer from this phenomenon. Consequently, a plume composed of many small puffs will evolve along the prevailing winds more accurately than plumes composed of puffs with significant extents. 
We proceed by presenting a survey of the related literature, the integral and Gaussian models, the fragmentation process, and experiments from our atmospheric plume simulator.

\section{Related Literature}

Several models of passive air dispersion exist, and they may be organized according to their level of mathematical complexity [2]. Among the typical classes of dispersion models, we find: gross screening models, intermediate models, and advanced models, as per their degree of mathematical sophistication.

Gross screening models are those that require the simplest tools and are generally used in the absence of more elaborate models to make worst-case predictions [2]. The sophistication of the input data is minimal and allows emergency planners to make crude estimations in a minimum of time. For instance, this class of models includes Hanna et al.'s work on the estimation of worst mean concentration downwind from a point source [5].

Intermediate models are able to take into account atmospheric stability classes and wind speed as inputs. This class is represented by models such as the diffusion equation, which describes smoke behaviour in turbulent air flows [6]. Analytical solutions to this diffusion equation include the Gaussian plume model in its simplest form for continuous point sources [3].

The Gaussian plume model is central to the modelling of atmospheric diffusion, and one of the remaining difficulties associated with its use is the determination of adequate diffusion coefficients (Gaussian standard deviations), which depend on local conditions at and around the site of release. This problem has been investigated since the first uses of this model. For instance, Barad was one of the first to determine adequate values for the diffusion parameters in prairie-like terrain conditions $[7,8]$. Based on this work, Pasquill and Gifford proposed coefficients for releases at low altitude over relatively obstacle-free terrains $[9,10]$. They examined the turbulence created by the heating of the atmosphere from the ground and defined atmospheric stability classes, for both daytime and night-time releases. While Pasquill and Gifford's results could be used over relatively flat terrains, McElory and Pooler contributed coefficient curves and tables for diffusion in urban environments which were much needed at the time [11].

Elaborate diffusion coefficients may also be derived when atmospheric conditions are known with precision. For instance, Draxler and others have related the values of the coefficients to velocity variation directly [12-14], to account for the increased turbulence and dispersion these fluctuations create.

Advanced models of passive diffusion include complex atmospheric phenomena such as plume ground reflection, elevated inversion, advection velocity, and plume rise with down-wash [2]. In the case of ground reflection, an elevated release will diffuse vertically until the bottom of the plume reaches the ground, where gas particles are reflected, or absorbed. In addition, as the plume elevates, it may reach an atmospheric temperature inversion, effectively dampening its ascension. When both phenomena are combined, the plume mostly remains in a layer comprised between the ground and the inversion. In practice, the concentration in the layer tends to reach uniformity, and can be estimated accordingly [3]. More recently, techniques have been designed to account for situations in which a plume is only partially reflected by an inversion [15].

From the ground to low altitudes, wind speed varies logarithmically and in turn influences plume velocity [16]. This effect is generally approximated with a power law, in which the value of the exponent is strongly influenced by the atmospheric stability class and the ground roughness, as noted by Irwin [17]. Atmospheric stability also has an effect on the mean wind profile and is taken into consideration by advanced passive diffusion models, using the Monin-Obukhov scale of meteorological parameters [5].

Initial release momentum and plume buoyancy generally cause a plume to rise rapidly, before prevalent winds make it bend in the downwind direction. Corrections have been suggested to include release momentum and buoyancy in advanced models by considering the effective height of the release, which varies with the intensity of the down-wash [18]. Simplifying assumptions, introduced by Davidson, must be posed to obtain closed-form equations for buoyant plumes [19].

While these techniques improve the performance of passive diffusion models, a problem subsists when the spatial extents of Gaussian puffs become so large as to encompass regions in which atmospheric conditions may vary substantially. Our research addresses this problem by establishing a Gaussian puff-breaking technique which may be employed to keep the maximum size of puffs as a constant, resulting in plumes composed of elements small enough to remain under consistent meteorological conditions.

\section{The Integral Model}

Once a dense gas is emitted, it enters a gravity flow stage where it collapses under its own weight for a period of time, until the ensuing entrainment of air (and possibly heating by solar radiation) dilutes the gas sufficiently to enter a passive dispersion stage. At this point, the cloud forms a layer which is in contact with the ground. Air flow then becomes the dominant factor involved in the dispersion of the cloud. The gravity flow stage for an instantaneous gas emission is generally modelled with a vertical gas cylinder whose properties, such as the atmospheric entrainment of air it creates, dimension, temperature, volume, and density may be estimated over time with the integral model. Fig. 1 shows such a cylinder undergoing gravitational collapse.

The radius of the cylinder corresponding to the instantaneous emission is expected to grow, as the gas cloud collapses under gravity. As this process takes place, the difference between the air and the gas densities diminishes to reach a point in time when the radius of the cylinder becomes stable [20] (at $\left.\rho=\rho_{a}\right)^{1}$ :

\footnotetext{
${ }^{1}$ A complete description of the variables and their units is given in Appendix A.
} 

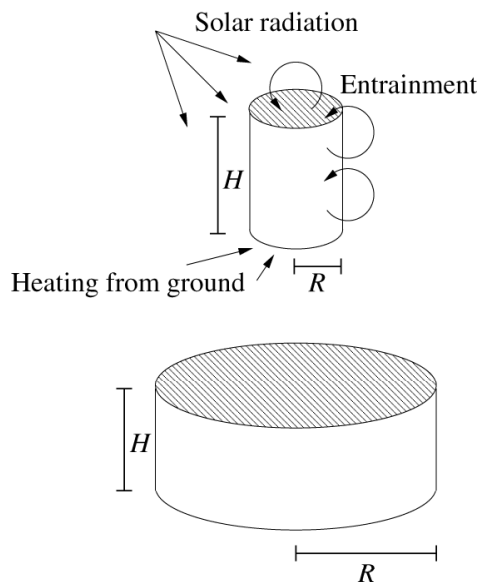

Figure 1. Collapse of gas cylinder under various environmental and gravitational effects.

$$
R^{\prime}=K \sqrt{g H \frac{\rho-\rho_{a}}{\rho_{a}}}
$$

The total mass of air entrained by the gas cloud, which significantly contributes to its dilution, evolves in time at a rate given by:

$$
M_{a}^{\prime}=\pi \rho_{a}\left(R^{\prime} 2 \alpha_{1} H R+R^{2} \alpha_{2} \frac{U_{t}}{R_{1}}\right)
$$

The entrainment of air is a function of the areas of the edge and top of the cylinder, as well as the speed of turbulent air.

The temperature of the cloud is influenced by various factors, the most significant being the temperature of the ground $\left(Q_{1}\right)$ with which the gas is in contact, and the temperature of the surrounding air $\left(Q_{2}\right)$ :

$$
T^{\prime}=\frac{Q_{1}+Q_{2}^{\prime}}{M_{a} C_{a}+M_{g} C_{g}}
$$

It is assumed that turbulent convection is the means by which heat is supplied to the gas from the ground:

$$
Q_{1}=\alpha_{3}\left(T-T_{s}\right)^{\frac{4}{3}}
$$

The heat transfer between the air and the cloud is expressed as:

$$
Q_{2}^{\prime}=M_{a}^{\prime} C_{a}\left(T_{a}-T\right)
$$

From previous equations, we can derive the volume, height, mean concentration, and density of the cloud:

$$
\begin{gathered}
V=\frac{M_{a}+M_{g}}{\rho} \\
H=\pi \frac{V}{R^{2}}
\end{gathered}
$$

$$
\begin{gathered}
C=\frac{M_{g}}{V} \\
\rho=T\left(M_{a}+M_{g}\right) T_{a}^{-1}\left(\frac{M_{a}}{\rho_{a}}+\frac{M_{g}}{\rho_{g}}\right)^{-1}
\end{gathered}
$$

The integral model is adequately suited for dense gas dispersions until the difference in gas and air densities becomes negligible. At this point, the model provides the initial parameters to a passive dispersion calculation, such as that carried out by a Gaussian model.

\section{The Gaussian Model}

The simplest form of atmospheric dispersion is passive. Nonetheless, determining the standard deviations for the Gaussian model in realistic cases remains a complex and experimental problem. According to Pasquill's experiments $[9,21]$, the initial standard deviations $\sigma_{y}$ and $\sigma_{z}$ (the crosswind and the vertical dispersion coefficients ${ }^{2}$, respectively) for the Gaussian model can be computed as:

$$
\sigma_{y} \text { or } \sigma_{z}=a x^{b}+c
$$

where $x$, the distance from the source, is expressed in kilometres. Values for parameters $a, b$, and $c$ are obtained from Tables 1 and 2, according to six atmospheric stability classes, from A (very unstable) to $\mathrm{F}$ (very stable). The values of these parameters differ whether $\sigma_{y}$ or $\sigma_{z}$ is computed. Atmospheric stability depends on factors such as wind speed, incident solar radiation, cloud cover, and possibly ground roughness at low altitudes. Tables 3 and 4 give the atmospheric stability class as a function of wind and solar radiation for daytime and as a function of wind and cloud cover for night-time. Once $\sigma_{x}, \sigma_{y}$, and $\sigma_{z}$ are computed and the simulation is ongoing, concentrations may be estimated at a given time step with the following Gaussian:

$C(\mathbf{x})=\frac{M}{(2 \pi)^{\frac{3}{2}} \operatorname{det} S} \exp \left\{-\frac{1}{2}\left[S^{-1}\left(\mathbf{x}-\mathbf{x}_{c}\right)\right]^{T} S^{-1}\left(\mathbf{x}-\mathbf{x}_{c}\right)\right\}$

where

$$
S=\left(\begin{array}{ccc}
\sigma_{x} & 0 & 0 \\
0 & \sigma_{y} & 0 \\
0 & 0 & \sigma_{z}
\end{array}\right)
$$

is the matrix of dispersion coefficients. Concentration is obtained at $\mathbf{x}=(x, y, z)^{T}$ while the centre of the Gaussian is located at $\mathbf{x}_{c}=\left(x_{c}, y_{c}, z_{c}\right)^{T}$. As the wind pushes the Gaussian cloud, its centre is updated with the prevailing wind velocity vector $\mathbf{u}$. In addition, the distance $x$ from the source to the centre of the Gaussian cloud is recomputed $\mathrm{as}^{3}$ :

\footnotetext{
${ }^{2}$ Pasquill assumed $\sigma_{x}=\sigma_{y}$, which is a reasonable hypothesis for crosswind dispersion.

3 The initial dispersion coefficients for the Gaussian are provided by the integral model after the gas cylinder has collapsed. Hence, the position of the source is said to be virtual, as it does not reflect the position of the gas cylinder.
} 
Table 1

Standard Deviations According to Pasquill's Atmospheric Stability Classes, for Passive Dispersions under $1 \mathrm{~km}$ [21]

\begin{tabular}{|l|c|c|c|c|}
\hline \multicolumn{5}{|c|}{$x \leq 1 \mathrm{~km}$} \\
\hline Class & $\sigma$ & $a$ & $b$ & $c$ \\
\hline $\mathrm{A}$ & $\sigma_{y}$ & 0.215 & 0.858 & 0.00 \\
& $\sigma_{z}$ & 0.467 & 1.890 & 0.01 \\
\hline $\mathrm{B}$ & $\sigma_{y}$ & 0.155 & 0.889 & 0.00 \\
& $\sigma_{z}$ & 0.103 & 1.110 & 0.00 \\
\hline $\mathrm{C}$ & $\sigma_{y}$ & 0.105 & 0.903 & 0.00 \\
& $\sigma_{z}$ & 0.066 & 0.915 & 0.00 \\
\hline $\mathrm{D}$ & $\sigma_{y}$ & 0.068 & 0.908 & 0.00 \\
& $\sigma_{z}$ & 0.032 & 0.822 & 0.00 \\
\hline $\mathrm{E}$ & $\sigma_{y}$ & 0.050 & 0.914 & 0.00 \\
& $\sigma_{z}$ & 0.023 & 0.745 & 0.00 \\
\hline $\mathrm{F}$ & $\sigma_{y}$ & 0.034 & 0.908 & 0.00 \\
& $\sigma_{z}$ & 0.014 & 0.727 & 0.00 \\
\hline
\end{tabular}

Table 2

Standard Deviations According to Pasquill's Atmospheric Stability Classes, for Passive Dispersions over $1 \mathrm{~km}$ [21].

Standard Deviations for Classes A through D are as per Table 1

\begin{tabular}{|l|c|c|c|r|}
\hline \multicolumn{5}{|c|}{$x>1 \mathrm{~km}$} \\
\hline Class & $\sigma$ & $a$ & $b$ & $c$ \\
\hline $\mathrm{E}$ & $\sigma_{y}$ & 0.050 & 0.914 & 0.00 \\
& $\sigma_{z}$ & 0.148 & 0.015 & -1.126 \\
\hline $\mathrm{F}$ & $\sigma_{y}$ & 0.034 & 0.908 & 0.000 \\
& $\sigma_{z}$ & 0.031 & 0.306 & -0.017 \\
\hline
\end{tabular}

Table 3

Daytime Atmospheric Stability Class as a Function of Wind and Solar Radiation

\begin{tabular}{|l|c|c|c|c|}
\hline \multirow{2}{*}{$\begin{array}{l}\text { Wind } \\
(\mathrm{m} / \mathrm{s})\end{array}$} & \multicolumn{4}{|c|}{ Solar Radiation $\left(\mathrm{W} \mathrm{m}^{2}\right)$} \\
\cline { 2 - 5 } & $\geq 925$ & $925-675$ & $675-175$ & $\leq 175$ \\
\hline$<2$ & A & A & B & D \\
\hline $2-3$ & A & B & C & D \\
\hline $3-5$ & B & B & C & D \\
\hline $5-6$ & C & C & D & D \\
\hline$>6$ & C & D & D & D \\
\hline
\end{tabular}

$$
x=\left(\frac{\sigma_{x}-c}{a}\right)^{\frac{1}{b}}
$$

This description of the Gaussian model accounts for one instantaneous emission of gas only. A source emit-
Table 4

Nighttime Atmospheric Stability Class as a Function of Wind and Cloud Cover

\begin{tabular}{|l|c|c|}
\hline \multirow{2}{*}{$\begin{array}{l}\text { Wind } \\
(\mathrm{m} / \mathrm{s})\end{array}$} & \multicolumn{2}{|c|}{ Cloud Cover $(\%)$} \\
\cline { 2 - 3 } & $\geq 50$ & $<50$ \\
\hline$<2$ & $\mathrm{~F}$ & $\mathrm{~F}$ \\
\hline $2-3$ & $\mathrm{E}$ & $\mathrm{F}$ \\
\hline $3-5$ & $\mathrm{D}$ & $\mathrm{E}$ \\
\hline$>5$ & $\mathrm{D}$ & $\mathrm{D}$ \\
\hline
\end{tabular}

Table 5

Values for $z_{0}^{\prime}$ Depend on Terrain Roughness

\begin{tabular}{|l|c|}
\hline Flat Terrain & $z_{0}^{\prime}=z_{0}$ \\
\hline Agricultural Land & $z_{0}^{\prime} \approx 0.10 \mathrm{~m}$ \\
\hline Garden Area & $z_{0}^{\prime} \approx 0.30 \mathrm{~m}$ \\
\hline Residential Area & $z_{0}^{\prime} \approx 1.00 \mathrm{~m}$ \\
\hline Urban Area & $z_{0}^{\prime} \approx 3.00 \mathrm{~m}$ \\
\hline
\end{tabular}

ting in a continuous fashion must be modelled differently. The instantaneous integral and Gaussian models can be extended to include series of instantaneous emissions over time, each emission at time $t_{i}$ possessing its own mass of gas $M_{i}$. Hence, to simulate a continuous emission, the integral model is fit with a gas cylinder stack while the Gaussian model receives a puff stack. Each new emission is placed in the gas cylinder stack, where the simulation begins. When a cylinder has reached relative stability, its parameters are fed into the puff stack of the Gaussian model, where it is left to develop according to prevailing environmental conditions. The resulting set of instantaneous emissions forms a plume governed by passive dispersion.

These models are obvious simplifications of environmental reality which entails more complex phenomena such as ground reflection and roughness, and particle fallout [4].

\subsection{Ground Roughness}

Variations in terrain quality, from plains to dense urban areas, affect the dispersion of gaseous puffs through increased turbulence. A correction, applied to the dispersion coefficients and introduced by Pasquill and Smith [21], amounts to computing the coefficient $\sigma_{z}$ as:

$$
\sigma_{z}=\left(a x^{b}+c\right)\left(\frac{z_{0}^{\prime}}{z_{0}}\right)^{0.53 x^{-0.22}}
$$

where $z_{0}^{\prime}$ is a measure of ground roughness expressed in $m$, and $z_{0}$ is a constant set to $0.03 \mathrm{~m}$. Table 5 indicates appropriate values for $z_{0}^{\prime}$ with respect to terrain characteristics. 


\subsection{Ground Reflection}

The ground may not entirely reflect gas particle fallouts, depending on gas and terrain properties. In light of this, a reflection correction is introduced, accounting for the resulting reduction in concentration as gas particles precipitate to the ground. This correction transforms the exponential in $z$ from (11) into:

$$
\exp \left\{-\frac{\left(z-z_{c}\right)^{2}}{2 \sigma_{z}^{2}}+\rho \frac{\left(z+z_{c}\right)^{2}}{2 \sigma_{z}^{2}}\right\}
$$

where $z_{c}$ is the emission height in metres and $\rho$ is ground reflection coefficient.

The rate of change in gaseous mass due to fallout can be obtained with:

$$
M^{\prime}=\frac{(r-1) M}{\sqrt{2 \pi}}\left(\frac{\sigma_{z}}{z^{2}+2 \sigma_{z}^{2}}+\frac{1}{\sqrt{2}} \tan ^{-1} \frac{z}{\sqrt{2 \sigma_{z}}}\right)
$$

evaluated between $z_{c}$ and $z_{c}+\frac{\left.\sigma_{z}\right|_{t+\delta t}}{\left.\sigma_{z}\right|_{t}}$. Coefficient $r$ represents ground reflection, which assumes values between 0.0 for no reflection, up to 1.0 for complete reflection.

\subsection{Model Interface}

There are several ways of combining the integral and the Gaussian model [21], and we adopted a method which poses the least number of hypotheses on the emission process. Once a gas cylinder has collapsed under gravity and its density has become similar to that of the surrounding air, the simulation must then turn to a passive mode, in the form of a 3D Gaussian puff. The interface resides in determining the initial parameters for the Gaussian from the integral model. At this critical stage, the gas mass must be conserved, and the parameters that require adjustments are the dispersion coefficients that are found in the covariance matrix $S$ of (11). Experiments have lead to the following relationships between the cylinder height and radius and the dispersion coefficients [4]:

$$
\begin{aligned}
R & =2.14 \sigma_{y} \\
H & =2.14 \sigma_{z}
\end{aligned}
$$

The initial position of the puff is given by the coordinates of the centre of the collapsed cylinder. In the passive mode of diffusion, the parameters requiring updating are the position of the puff, its mass (due to fallout), and its dispersion coefficients, from environmental variables such as ground roughness, reflection, direction and speed of prevailing winds, and so on.

\section{Puff Fragmentation}

The Gaussian model, when used to predict continuous emissions, possesses a relative adaptability as each puff evolves with respect to its local environmental conditions, provided that these are available on such a local scale. Over time, the scale of Gaussian puffs increases to a point

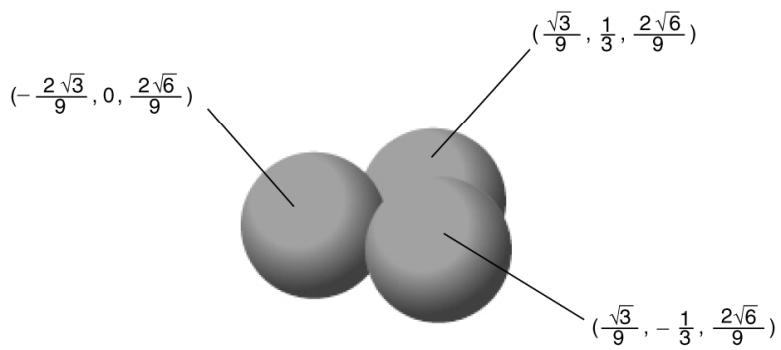

Figure 2. The positional symmetries of the top layer puffs starting at the bottom centre of the initial cloud.

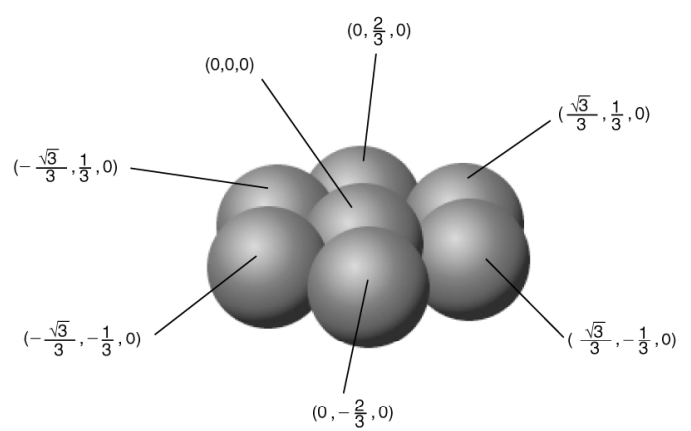

Figure 3. The positional symmetries of the middle layer puffs starting at the bottom centre of the initial cloud.

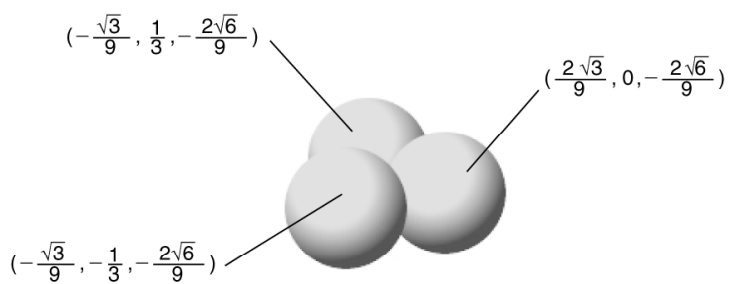

Figure 4. The positional symmetries of the bottom layer puffs starting at the bottom centre of the initial cloud.

where atmospheric conditions within their extent may vary significantly. To account for such variation, an effective approach consists of fragmenting large Gaussian puffs into smaller ones, while preserving the properties of the plume, such as spread and concentration. The original Gaussian puff prior to the fragmentation is represented by an elliptical sphere. We use a hexagonal sphere packing scheme to create a group of elliptical spheres, each a Gaussian puff, with a distribution as close to the original Gaussian as possible. This fragmentation ensures a minimal number of elliptical spheres with relative positional symmetries. Figs. $2-4$ show such a fragmentation, along with positional symmetries of the sphere centres for an initial Gaussian puff centreed at coordinates $\mathbf{x}_{c}=(0,0,0)^{T}$. 


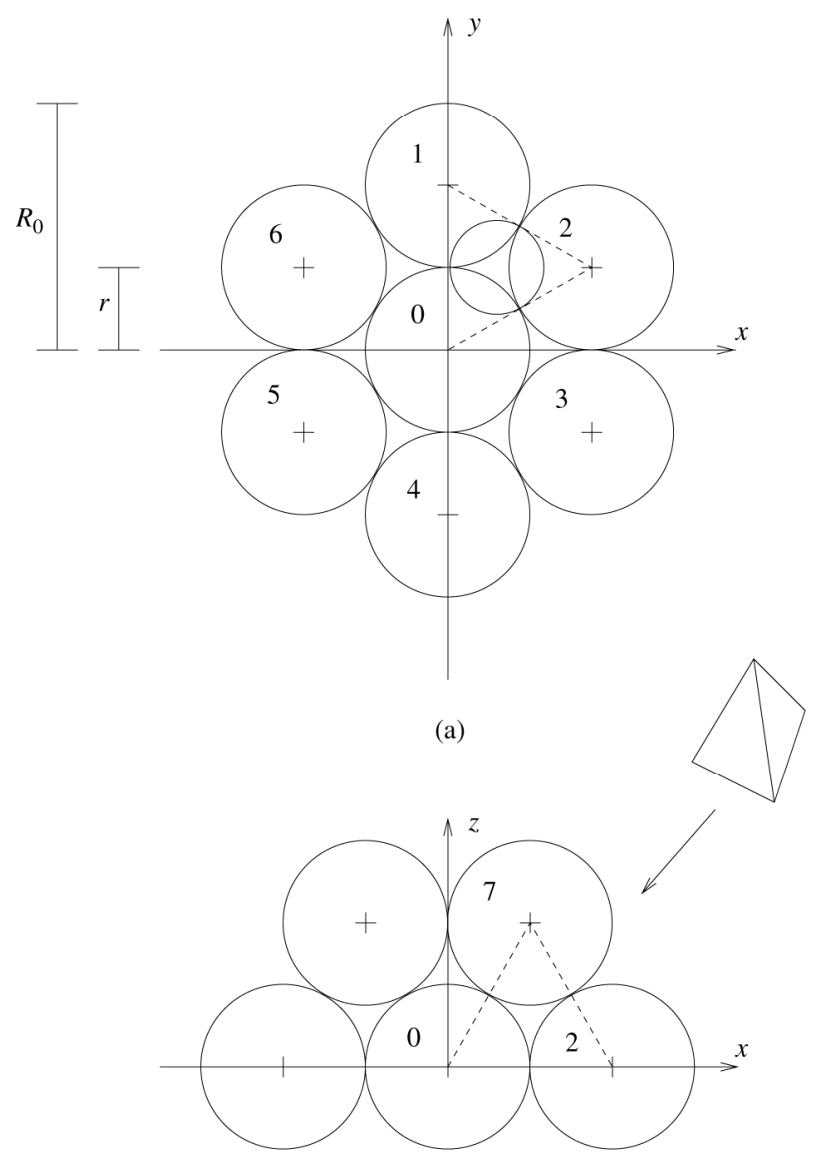

(b)

Figure 5. The computation of sphere centres. (a) The radius of the inscribed equilateral triangle formed by the centres of spheres $\mathbf{r}_{0}, \mathbf{r}_{1}$, and $\mathbf{r}_{2}$, yields the $x, y$ coordinates of sphere $\mathbf{r}_{7}$ from the top layer and (b) the height of the tetrahedron formed by the centres of spheres $\mathbf{r}_{0}, \mathbf{r}_{1}, \mathbf{r}_{2}$, and $\mathbf{r}_{7}$ yields the $z$ coordinate of sphere $\mathbf{r}_{7}$.

\subsection{Computing Gaussian Sphere Centres}

Let $C(\mathbf{x})$ be a 3D Gaussian with covariance matrix $S$, as in (11). After fragmentation into a hexagonal packing, the 13 resulting Gaussians have covariance matrices $S_{f}=\frac{2}{3} S$ with the centre coordinates of the centre Gaussian set to $\mathbf{r}_{0}=(0,0,0)^{T}$. The $x, y$ plane contains the centres of the six Gaussians surrounding $\mathbf{r}_{0}$, labeled clockwise from $\mathbf{r}_{1}$ to $\mathbf{r}_{6}$ starting with the top one on the $y$-axis, as per Fig. 5(a).

The centre coordinates of Gaussian $\mathbf{r}_{1}$ are immediately obtained as: $\mathbf{r}_{1}=S\left(0, \frac{2}{3}, 0\right)^{T}$. The rightmost point of the dotted equilateral triangle with side $2 r$ (or $\frac{2}{3}$ ) shown in Fig. 5(a) yields the $x, y$ coordinates of Gaussian $\mathbf{r}_{2}$. The $y$ coordinate is equal to $r$ (or $\frac{1}{3}$ ), and the $x$ coordinate is obtained as $x^{2}+r^{2}=(2 r)^{2}$. Hence, $x=\frac{\sqrt{3}}{3}$ and the centre of $\mathbf{r}_{2}$ is $S\left(\frac{\sqrt{3}}{3}, \frac{1}{3}, 0\right)^{T}$. The centre coordinates of $\mathbf{r}_{3}$ and $\mathbf{r}_{4}$ are obtained by folding around the $x$-axis as $\mathbf{r}_{3}=S\left(\frac{\sqrt{3}}{3}, \frac{-1}{3}, 0\right)^{T}$ and $\mathbf{r}_{4}=S\left(0, \frac{-2}{3}, 0\right)$. The centre coordinates of $\mathbf{r}_{5}$ and $\mathbf{r}_{6}$ are obtained by folding around the $y$-axis as $\mathbf{r}_{5}=S\left(\frac{-\sqrt{3}}{3}, \frac{-1}{3}, 0\right)^{T}$ and $\mathbf{r}_{6}=S\left(\frac{-\sqrt{3}}{3}, \frac{1}{3}, 0\right)$.
The top layer consists of three Gaussian spheres that are packed hexagonally from the central layer, as in Fig. 5(b). The first sphere $\mathbf{r}_{7}$ is in contact with central layer spheres $\mathbf{r}_{0}, \mathbf{r}_{1}$, and $\mathbf{r}_{2}$ and its $x$ centre coordinate is given by the radius of the inscribed circle inside the dotted triangle: $\frac{\sqrt{3}}{9}$. As the sides of the triangle are $2 r$ in length, the $y$ coordinate is given by $r$ or $\frac{1}{3}$. The $z$ coordinate can be obtained as the height of the tetrahedron of side $2 r$ formed by the centres of Gaussian spheres $\mathbf{r}_{0}, \mathbf{r}_{1}, \mathbf{r}_{2}$, and $\mathbf{r}_{7}$ as $\frac{2 \sqrt{6}}{9}$ (depicted in Fig. 5(b)). Hence, the coordinates of $\mathbf{r}_{7}$ are $S\left(\frac{\sqrt{3}}{9}, \frac{1}{3}, \frac{2 \sqrt{6}}{9}\right)^{T}$. Progressing clockwise, the second sphere $\mathbf{r}_{8}$ of the top layer is in contact with spheres $\mathbf{r}_{0}, \mathbf{r}_{3}$, and $\mathbf{r}_{4}$ from the central layer, and its centre coordinates are obtained by folding the $x$-axis: $\mathbf{r}_{8}=S\left(\frac{\sqrt{3}}{9}, \frac{-1}{3}, \frac{2 \sqrt{6}}{9}\right)^{T}$. Sphere $\mathbf{r}_{9}$ is in contact with spheres $\mathbf{r}_{0}, \mathbf{r}_{5}$, and $\mathbf{r}_{6}$ of the central layer. Due to its placement, the $y$ coordinate of the centre of this sphere is simply 0 . In addition, because the three Gaussian spheres from the top layer are equidistant from the point $(x, y)=(0,0)$, its $x$ coordinate is obtained as the negative of the length of the segment from $(x, y)=(0,0)$ to the centre of sphere $\mathbf{r}_{7}$, projected onto the $x, y$ plane: $\frac{-2 \sqrt{3}}{9}$. Hence, $\mathbf{r}_{9}=S\left(\frac{-2 \sqrt{3}}{9}, 0, \frac{2 \sqrt{6}}{9}\right)^{T}$. Fig. 6(a) shows the final placement of the Gaussian spheres from the top layer.

As there are only two ways of placing the top or the bottom layer with respect to the central one, we chose to place the bottom layer in a different configuration from the top layer, to achieve symmetry of concentration in the Gaussian sphere pack. To this end, sphere $\mathbf{r}_{10}$ is placed such as to create contact with spheres $\mathbf{r}_{0}, \mathbf{r}_{1}$, and $\mathbf{r}_{6}$ from the central layer. Its centre coordinates are immediately obtained by symmetry as $\mathbf{r}_{10}=S\left(\frac{-\sqrt{3}}{9}, \frac{1}{3}, \frac{-2 \sqrt{6}}{9}\right)^{T}$. The centres for $\mathbf{r}_{11}$ and $\mathbf{r}_{12}$ are obtained symmetrically as $\mathbf{r}_{11}=S\left(\frac{2 \sqrt{3}}{9}, 0, \frac{-2 \sqrt{6}}{9}\right)^{T}$, and $\mathbf{r}_{12}=S\left(\frac{-\sqrt{3}}{9}, \frac{-1}{3}, \frac{-2 \sqrt{6}}{9}\right)^{T}$. Fig. 6(b) shows the final placement of the spheres from the bottom layer, while Figs. 2-4 show the positional symmetries of the Gaussian sphere pack.

\subsection{Error Minimization}

This Gaussian puff fragmentation scheme must maintain essential properties such as the conservation of both the total gaseous mass and the geographical distribution of concentrations. To this end, our initial, empirically determined approximation consists of a central puff containing $21.81 \%$ of the initial gaseous mass with the remaining 12 puffs containing $6.52 \%$ each. The dispersion coefficients $\sigma_{x}, \sigma_{y}$, and $\sigma_{z}$ from each of the 13 puffs are set to $\frac{2}{3}$ of the initial cloud coefficients, while the sphere centres are set to $k_{f} \mathbf{r}_{i}$ :

$$
\begin{aligned}
C= & \frac{M}{(2 \pi)^{\frac{3}{2}} \operatorname{det} S} \exp \left\{-\frac{1}{2}\left[S^{-1}\left(\mathbf{x}-\mathbf{x}_{c}\right)\right]^{T} S^{-1}\left(\mathbf{x}-\mathbf{x}_{c}\right)\right\} \\
\approx & \sum_{i=0}^{12} \frac{M_{i}}{(2 \pi)^{\frac{3}{2}} \operatorname{det} S_{f}} \exp \left\{-\frac{1}{2}\left[S_{f}^{-1}\left(\mathbf{x}-\mathbf{x}_{c}-k_{f} \mathbf{r}_{i}\right)\right]^{T}\right. \\
& \left.S_{f}^{-1}\left(\mathbf{x}-\mathbf{x}_{c}-k_{f} \mathbf{r}_{i}\right)\right\}
\end{aligned}
$$




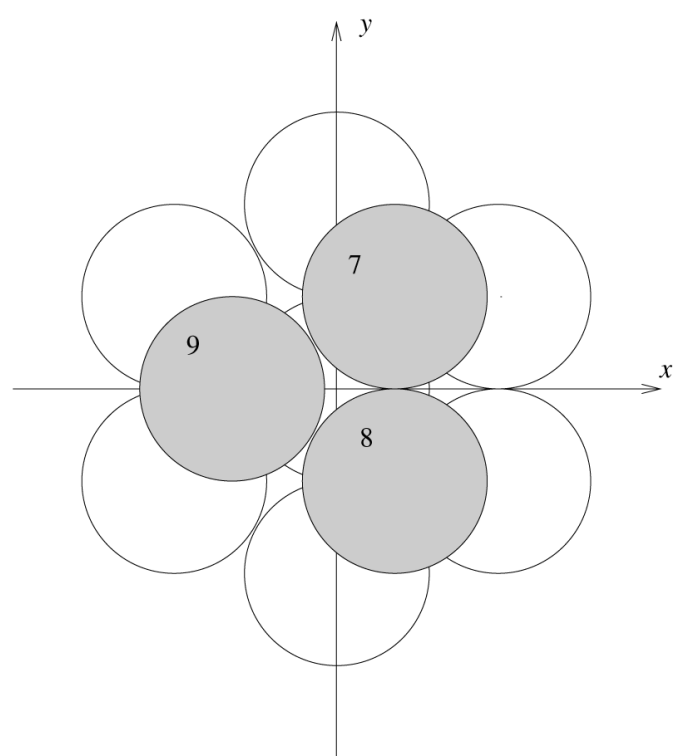

(a)

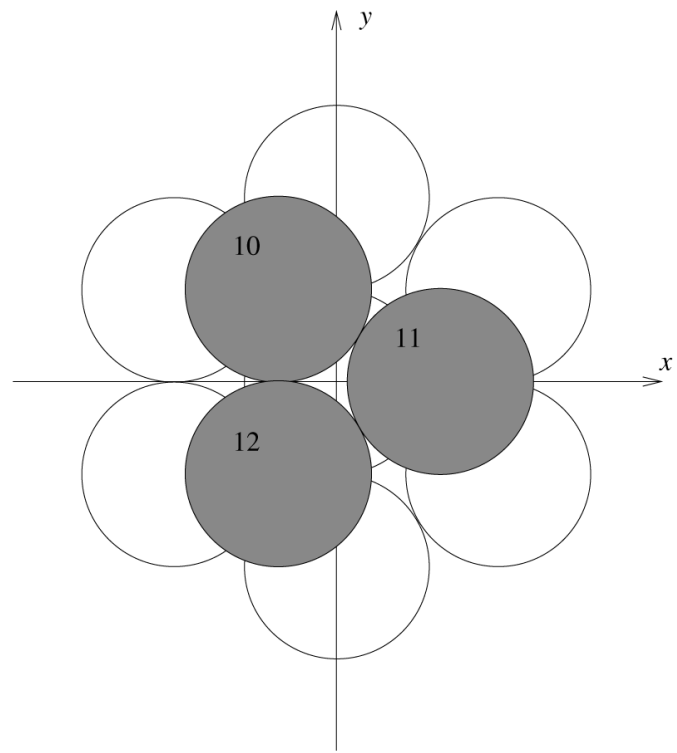

(b)

Figure 6. (a) The top layer sphere arrangement and (b) the bottom layer sphere arrangement.

where $S_{f}=k_{\sigma} S, k_{\sigma}=\frac{2}{3}, k_{f}=\pi^{\frac{2}{3}}$,

$$
M_{i}= \begin{cases}0.218107 & \text { if } i=0 \\ 0.065158 & \text { otherwise }\end{cases}
$$

and

$$
\begin{aligned}
& \mathbf{r}_{0}=S(0,0,0)^{T} \\
& \mathbf{r}_{1}=S\left(0, \frac{2}{3}, 0\right)^{T} \\
& \mathbf{r}_{2}=S\left(\frac{\sqrt{3}}{3}, \frac{1}{3}, 0\right)^{T}
\end{aligned}
$$

$$
\begin{aligned}
\mathbf{r}_{3} & =S\left(\frac{\sqrt{3}}{3}, \frac{-1}{3}, 0\right)^{T} \\
\mathbf{r}_{4} & =S\left(0, \frac{-2}{3}, 0\right)^{T} \\
\mathbf{r}_{5} & =S\left(\frac{-\sqrt{3}}{3}, \frac{-1}{3}, 0\right)^{T} \\
\mathbf{r}_{6} & =S\left(\frac{-\sqrt{3}}{3}, \frac{1}{3}, 0\right)^{T} \\
\mathbf{r}_{7} & =S\left(\frac{\sqrt{3}}{9}, \frac{1}{3}, \frac{2 \sqrt{6}}{9}\right)^{T} \\
\mathbf{r}_{8} & =S\left(\frac{\sqrt{3}}{9}, \frac{-1}{3}, \frac{2 \sqrt{6}}{9}\right)^{T} \\
\mathbf{r}_{9} & =S\left(\frac{-2 \sqrt{3}}{9}, 0, \frac{2 \sqrt{6}}{9}\right)^{T} \\
\mathbf{r}_{10} & =S\left(\frac{-\sqrt{3}}{9}, \frac{1}{3}, \frac{-2 \sqrt{6}}{9}\right)^{T} \\
\mathbf{r}_{11} & =S\left(\frac{2 \sqrt{3}}{9}, 0, \frac{-2 \sqrt{6}}{9}\right)^{T} \\
\mathbf{r}_{12} & =S\left(\frac{-\sqrt{3}}{9}, \frac{-1}{3}, \frac{-2 \sqrt{6}}{9}\right)^{T}
\end{aligned}
$$

While approximation (18) preserves the total gas mass, the distribution of concentrations still slightly varies from the initial Gaussian puff, as shown by the error function in Fig. 7. Hence, we defined a functional to be minimized with respect to $k_{\sigma}$ and $k_{f}$, which are the parameters that influence the distribution of concentration the most:

$$
\begin{aligned}
F\left(k_{\sigma}, k_{f}\right)= & \frac{1}{(2 \pi)^{\frac{3}{2}}} \int_{-\infty}^{\infty}\left[\frac { M } { \operatorname { d e t } S } \operatorname { e x p } \left\{-\frac{1}{2}\left[S^{-1}\left(\mathbf{x}-\mathbf{x}_{c}\right)\right]^{T}\right.\right. \\
& \left.S^{-1}\left(\mathbf{x}-\mathbf{x}_{c}\right)\right\} \\
& -\sum_{i=0}^{12} \frac{M_{i}}{\operatorname{det} S_{f}} \exp \left\{-\frac{1}{2}\left[S_{f}^{-1}\left(\mathbf{x}-\mathbf{x}_{c}-k_{f} \mathbf{r}_{i}\right)\right]^{T}\right. \\
& \left.\left.S_{f}^{-1}\left(\mathbf{x}-\mathbf{x}_{c}-k_{f} \mathbf{r}_{i}\right)\right\}\right]^{2} d \mathbf{x}
\end{aligned}
$$

We used Polak-Ribiere's conjugate-gradient descent method to minimize (19) with respect to $k_{\sigma}$ and $f_{f}$. The procedure lead to the following values: $k_{\sigma}=0.754529$ and $k_{f}=1.830323^{4}$. The resulting error function displayed in Fig. 8 evaluated to $4.01 \mathrm{E}^{-4}$ and shows the improvement over our initial approximation, illustrated in Fig. 8.

\footnotetext{
4 The choice of keeping the mass distribution constant over the minimization process is motivated by the fact that the inclusion of these terms invariably leads to a central fragmented Gaussian sharing its parameters with the initial one (mass and dispersion coefficients), and a zero mass for the remaining fragmented Gaussians.
} 


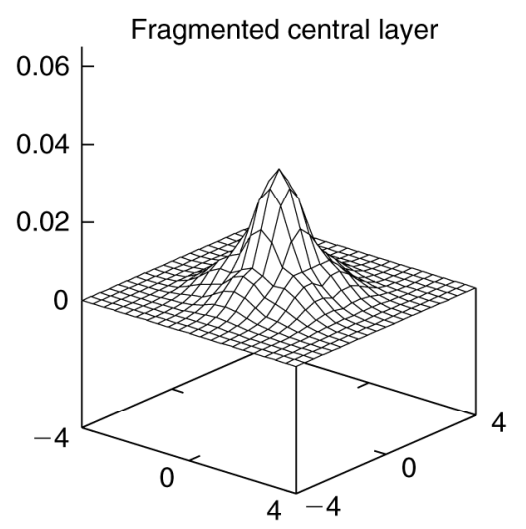

(a)

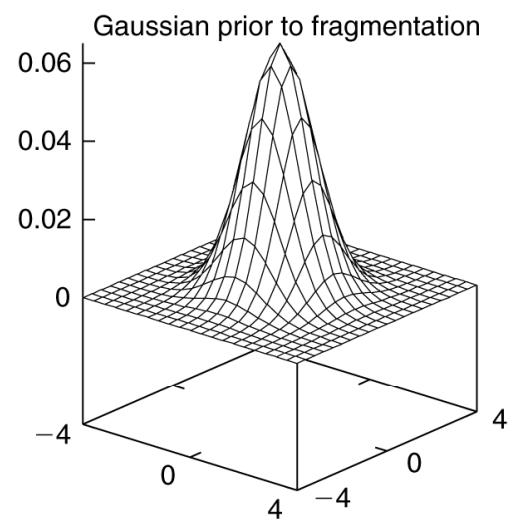

(d)

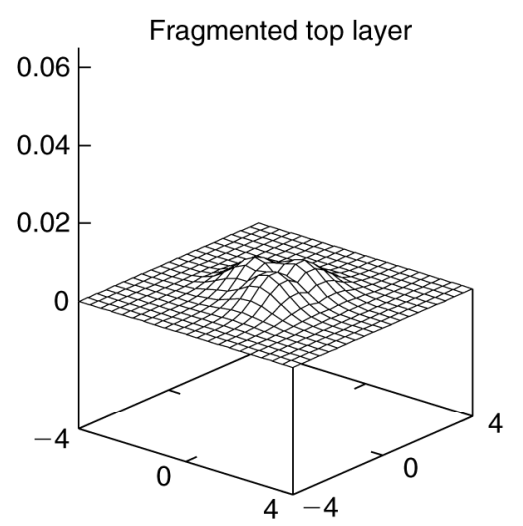

(b)

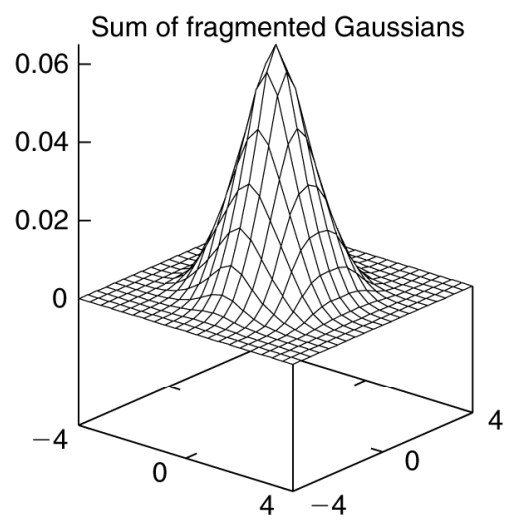

(e)

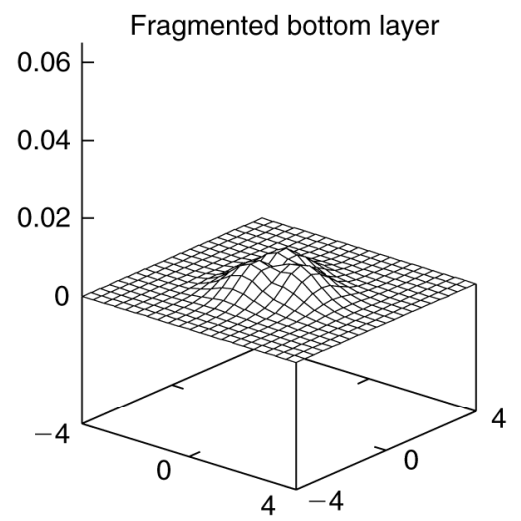

(c)

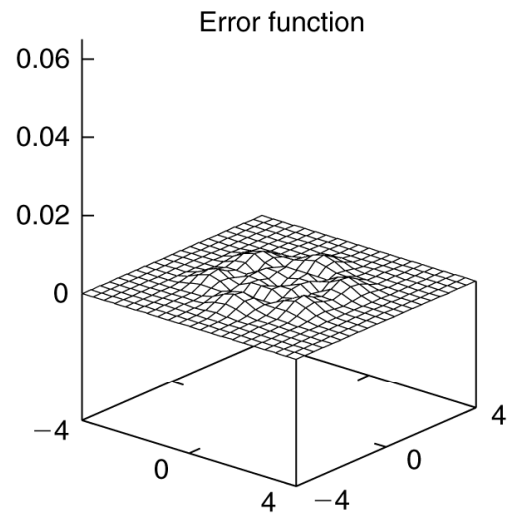

(f)

Figure 7. The Gaussian fragmentation process using our initial parameters $k_{\sigma}$ and $k_{f}$. The 13 Gaussians generated after fragmentation of the initial Gaussian: (a) the centre layer, (b) the top layer, (c) the bottom layer, (d) the Gaussian prior to fragmentation, followed by (e) the sum of the 13 Gaussians, and (f) the error function.

Gaussian prior to fragmentation

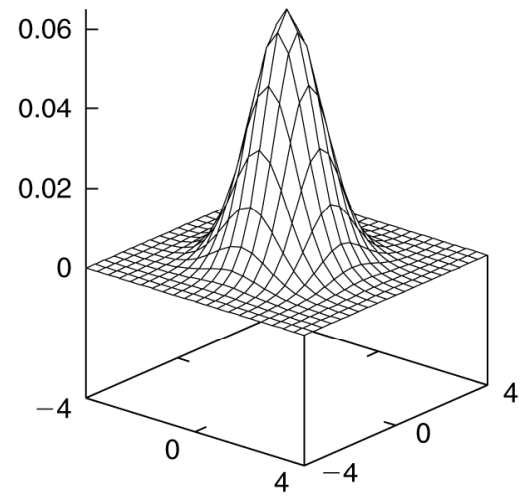

(a)
Sum of fragmented Gaussians

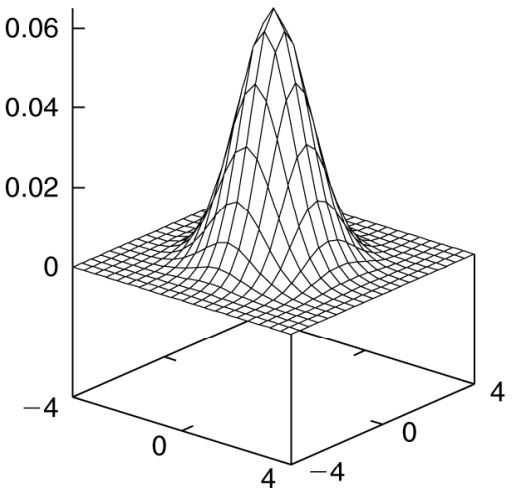

(b)

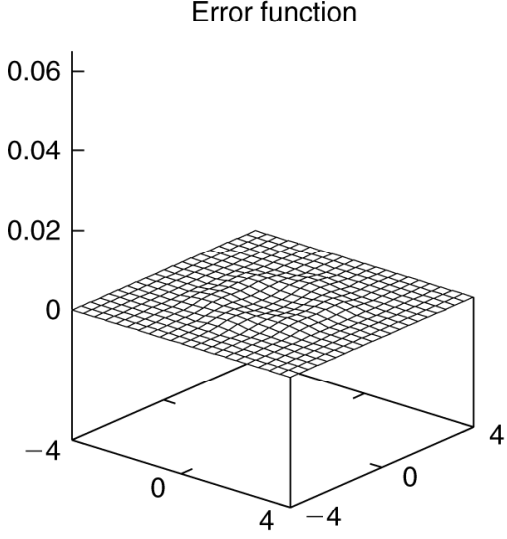

(c)

Figure 8. The results obtained with the minimization of the error functional: (a) the Gaussian prior to fragmentation, followed by (b) the sum of the 13 Gaussians, and (c) the error functional.

\section{Parallelization}

From a computational standpoint, the combined integralGaussian model with puff fragmentation is demanding, particularly when the number of Gaussian puffs becomes large. Fortunately, the model lends itself rather naturally to parallelization.

A number of observations concerning the characteristics of the combined model can be made: during a simulation, the integral model is in use for what amounts to be 


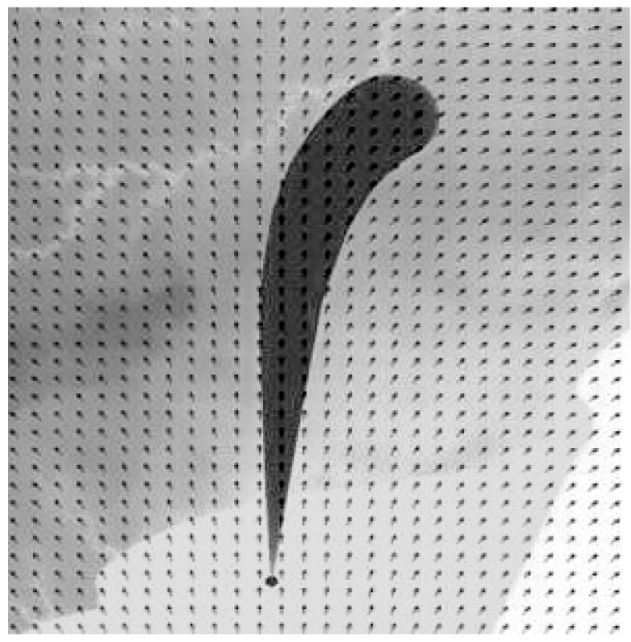

(a)

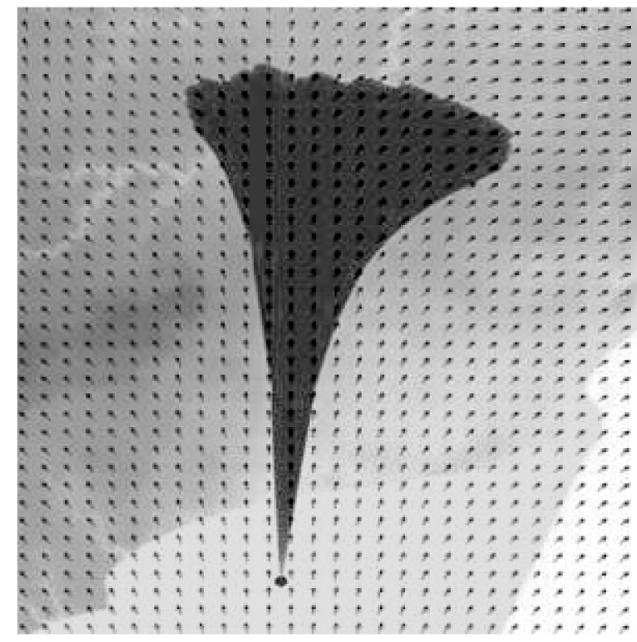

(b)

Figure 9. (a) Classical plume progression from a continuous emission source and (b) plume progression with Gaussian puff fragmentation. Different trajectories under identical wind vector fields are experienced.

a small duration per emitted gas cylinder, owing to rapid collapse and dilution. In comparison, the Gaussian model is computationally costlier, due to the number of Gaussian puffs fed to its stack by the integral model, the duration of each puff (hours, perhaps days), and particularly the puff fragmentation mechanism, which exponentially increases the number of puffs entering the simulation. In addition, one of the most demanding processes is the calculation of estimated concentrations over grid maps.

Consider a continuous gaseous emission lasting a total time $T=N_{1} t$, with a Gaussian puff emitted at every $t$. The total number of puffs, without fragmentation is thus $N_{1}$. If we assume that each puff is fragmented $n$ times, then $N$, the number of Gaussian puffs in the simulation is:

$$
N=N_{1} \sum_{i=0}^{n} P^{i}
$$

where $P=12$. For instance, if 1,000 initial puffs are released, after four simultaneous fragmentations, the number of puffs in the simulation reaches $22,621,000$. In addition, numerous concentration calculations must be carried out for each puff, and the amount of these increases as the extent of the puffs becomes larger. A monoprocessor architecture is clearly inadequate for long simulations such as radiological emissions and volcanic phenomena, which may last for days.

An adequate parallel architecture for the parallelization of the combined integral-Gaussian model is a sharedmemory, multiprocessor architecture allowing computing units to share one instance of the grid map of the region of interest, while ensuring an adequate distribution of the computation of dispersion and concentration. For instance, the puffs could be evenly distributed among available processors in a natural fashion each time they are introduced in the simulation. In the context of the preceding example, a multiprocessor architecture with $2^{10}$ available computing units would, at the peak of the simulation, reduce the com- puting load from 22 million puffs to 22,091 per processor, which is an acceptable computational burden.

\section{Experiments}

Two sets of experiments were conducted to demonstrate the extended capabilities the fragmented Gaussian plume system possesses.

The first set of simulation experiments were conducted over an elevation grid map of the Sarnia region in Ontario, Canada (see Figs. 9 and 10). Elevation is colour-coded from yellow (low elevation) to orange (high elevation). The Gaussian puffs forming the gaseous plumes are displayed with transparency factors unrelated to concentration, to show their position. The extent of the displayed puffs is a fraction of their dispersion coefficients. These experiments are conducted with average atmospheric conditions (Pasquill's stability class D) for $1.5-3 \mathrm{~h}$ of real dispersion time. Wind direction is variable with speed $\|\mathbf{u}\|_{2}$ averaging $1.5 \mathrm{~m} / \mathrm{s}$. The gas emission $M_{g}$ is set to $0.005 \mathrm{~kg}$ and occurs at every second. The simulation time step $\delta t$ is set to $10 \mathrm{~s}$. A list for the values of the remaining parameters can be found in Appendix B.

The first experiment displayed in Fig. 9 demonstrates, under a spatially variable wind vector field (divergent, in the north direction), the difference in gaseous progression between the classical integral-Gaussian plume model with and without puff fragmentation. Fig. 9(a) shows the dispersion results when the extent of puffs becomes sufficiently large to be subjected to more than a single wind vector: the plume deviates towards the dominant wind. However, as shown in Fig. 9(b), with a puff fragmentation mechanism, the trajectory of the plume is consonant with the variation observed in the wind vector field, resulting in an improvement in the realism of the plume progression [22].

The second experiment, shown in Fig. 10, is a sequence of images showing the puff fragmentation mechanism. The blue region represents the emission site, simulated with the integral model. In Fig. 10(a) the initial gaseous emission 


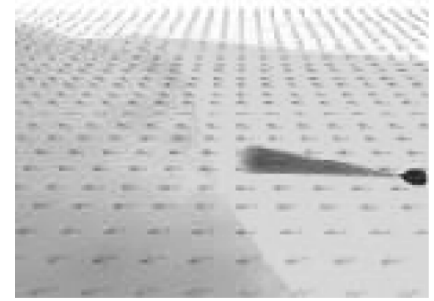

(a)

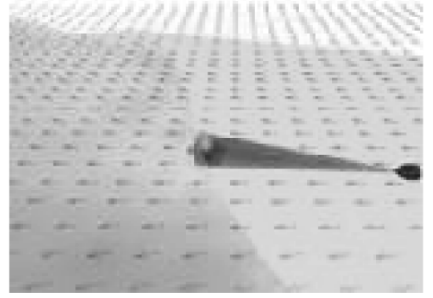

(b)

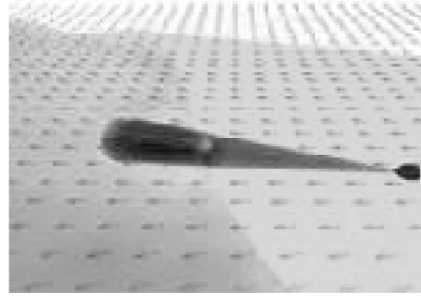

(c)

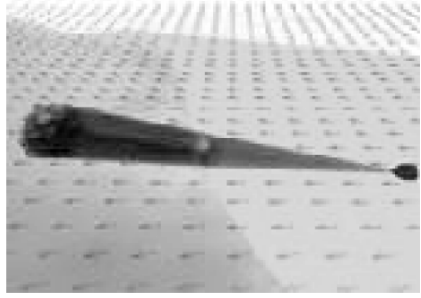

(d)

Figure 10. A sequence of images showing the Gaussian puff fragmentation, occurring at puff radii reaching $500 \mathrm{~m}$ : (a) the initial puff progression, (b) the first fragmentation occurrence, (c) the progression of the resulting puffs, and (d) the second fragmentation occurrence.

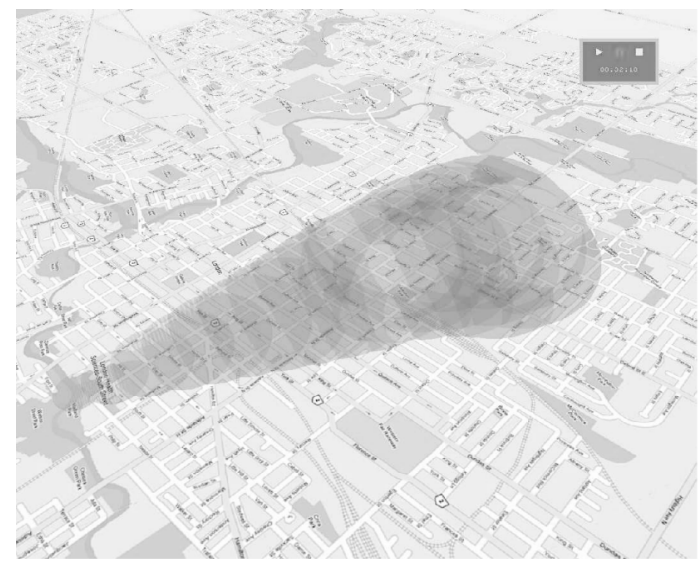

(a)

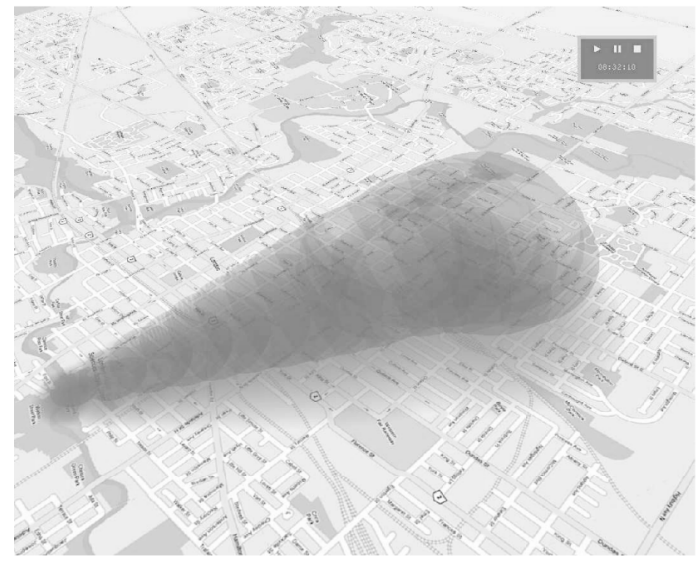

(c)

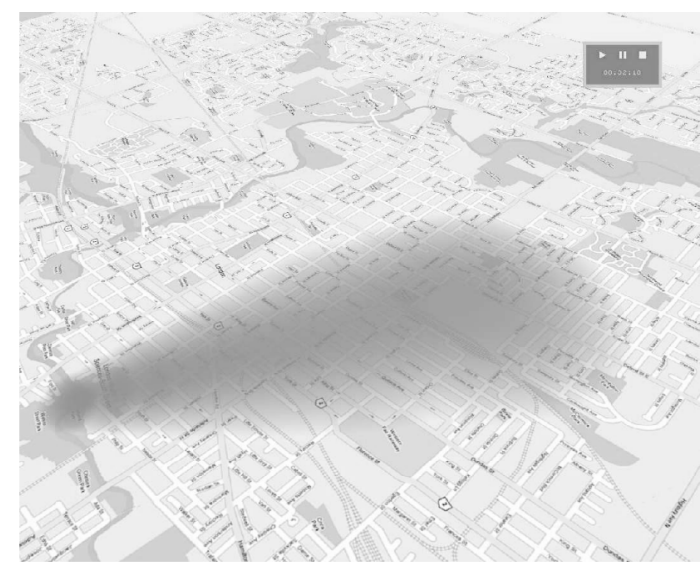

(b)

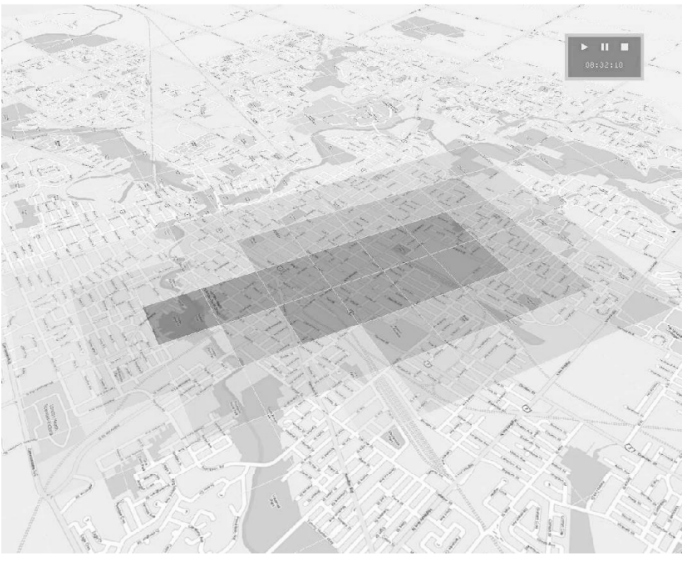

(d)

Figure 11. (a) A typical plume with Gaussian fragmentation over a populated area, (b) its colour-coded ground-level concentration levels, (c) the plume superimposed onto the concentration levels, and (d) an evacuation zone related to the gaseous release.

starts with a single Gaussian puff. After an amount of time into the simulation, the largest dispersion coefficient $\left(\sigma_{x}\right.$, $\sigma_{y}$, or $\sigma_{z}$ ) reaches $500 \mathrm{~m}$, and triggers the fragmentation process. The resulting puffs are displayed in Fig. 10(b). As time elapses, the puffs experience progression and increased extent, as in Fig. 10(c). Ultimately, the fragmentation process is triggered a second time, as depicted in Fig. 10(d).

The second set of experiments uses an urban map of the regional city of London, Ontario, in Canada, and is designed to illustrate the capabilities of the fragmented plume model. Atmospheric conditions for this experiment consist of light northern winds $(0.4 \mathrm{~m} / \mathrm{s})$, atmospheric stability class $\mathrm{D}$, and $8.0 \mathrm{~h}$ of real-time dispersion. Unless indicated otherwise, other simulation parameters are as in Appendix B.

Fig. 11(a) displays a plume that has undergone Gaus- 
sian fragmentation after 3:57 h of real-time dispersion. The Gaussian puffs are displayed using translucent 3D elliptical spheres with dimensions consonant with the dispersion coefficients of the Gaussian puffs. Fig. 11(b) displays the concentrations of the plume at ground level. The absence of discontinuities in the concentration spread is due to the successful minimization of error functional (19). Fig. 11(c) displays the 3D plume over the estimated concentration at ground level, while Fig. 11(d) is a putative warning and evacuation zone upon this simulated gaseous release. This zone varies depending on the chemical being released and the prevailing environmental conditions.

Our experiments qualitatively demonstrate the extended capabilities of the fragmented Gaussian plume model against traditional dispersion techniques, when local meteorological conditions vary significantly over large spatial extents (as in Fig. 9). Our model is effective against highly variable wind conditions that account for the imprecisions noted in more classical plume models which are all exempt of fragmentation.

\section{Conclusion}

Air dispersion modelling remains an elusively imprecise branch of environmental science [22]. Inherent difficulties are numerous, among which we find the experimental evaluation of various critical parameters, such as Pasquill's dispersion coefficients [21], or the mathematical complexities involved with modelling turbulent phenomena.

We proposed a combined integral-Gaussian atmospheric dispersion model including a mechanism for the fragmentation of gas puffs. The immediate benefits of this model reside in the improvement in concentration and spread predictions under variable wind conditions, at an increased computational cost, which may be alleviated by an adequate parallel implementation on shared-memory, massively parallel computing equipment. This accurate gas plume technique has been adopted by emergency planners and the resulting algorithms are currently being included in commercial software. Improvements to this model include the inclusion of light gases, and adequate model corrections for terrains with significant slopes and obstacles, in the case of dense gas emissions.

\section{A. Notation}

$$
\begin{aligned}
& C: \quad \text { gas concentration }\left(\mathrm{kg} \mathrm{m}^{3}\right) \\
& C_{a}: \text { specific heat of air }(\mathrm{kg} \mathrm{J} / \mathrm{K}) \\
& C_{g}: \text { specific heat of gas }(\mathrm{kg} \mathrm{J} / \mathrm{K}) \\
& H: \text { height of gas cylinder }(\mathrm{m}) \\
& K: \quad \text { Van Ulden's parameter }(\mathrm{m}) \\
& M: \text { mass of pollutant }(\mathrm{kg}) \\
& M_{a}: \text { air entrained by cylinder }(\mathrm{kg}) \\
& M_{g}: \text { mass of gas in cylinder }(\mathrm{kg}) \\
& Q_{1}: \text { heating by the ground }(\mathrm{J})
\end{aligned}
$$

$Q_{2}$ : heating by the air $(\mathrm{J})$

$R$ : radius of gas cylinder $(\mathrm{m})$

$R_{1}$ : Richardson's number

$T: \quad$ gas temperature $(\mathrm{K})$

$T_{a}$ : air temperature $(\mathrm{K})$

$T_{s}$ : ground temperature $(\mathrm{K})$

$U_{t}$ : horizontal turbulent air speed $(\mathrm{m} / \mathrm{s})$

$V:$ cylinder volume $\left(\mathrm{m}^{3}\right)$

$g: \quad$ gravity $\left(\mathrm{m} / \mathrm{s}^{2}\right)$

$\alpha_{1}$ : air entrainment by cylinder edge

$\alpha_{2}$ : air entrainment by cylinder top

$\alpha_{3}$ : gas thermal conductivity ( $\left.\mathrm{JK}^{\frac{3}{4}}\right)$

$\rho: \quad$ average cylinder density $\left(\mathrm{kg} / \mathrm{m}^{3}\right)$

$\rho_{a}:$ air density $\left(\mathrm{kg} / \mathrm{m}^{3}\right)$

u: $(u, v)^{T}$ wind velocity $(\mathrm{m} / \mathrm{s})$

$\mathbf{x}: \quad(x, y, z)^{T}$ point of concentration

$\mathbf{x}_{c}:\left(x_{c}, y_{c}, z_{c}\right)^{T}$ Gaussian centre

\section{B. Experimental Values}

$$
\begin{array}{ll}
C_{a}: & 1,000 \mathrm{~J} / \mathrm{kg} / \mathrm{K} \\
C_{g}: & 2,400 \mathrm{~J} / \mathrm{kg} / \mathrm{K} \\
K: & 1 \\
M_{a}: & 0.4 \mathrm{~kg} \\
R_{1}: & 1 \\
T: & 300 \mathrm{~K} \\
T_{a}: & 295 \mathrm{~K} \\
T_{s}: & 293 \mathrm{~K} \\
U_{t}: & 1 \mathrm{~m} / \mathrm{s} \\
g: & 9.81 \mathrm{~N} / \mathrm{kg} \\
\rho_{a}: & 1.184 \mathrm{~kg} / \mathrm{m}^{3} \\
\rho_{g}: & 2.4 \mathrm{~kg} / \mathrm{m}^{3}
\end{array}
$$

\section{References}

[1] M. Benarie, Urban air pollution modeling (Cambridge, MA: MIT Press, 1980).

[2] R. MacDonald, Theory and objectives of air dispersion modeling, Internal Report MME 474, University of Waterloo, Waterloo, Ontario, Canada, 2003.

[3] D.B. Turner, Workbook of atmospheric dispersion estimates: An introduction to air dispersion modeling, Second Edition (Boca Raton, FL: CRC Press, 1994).

[4] European Process Safety Center, Atmospheric dispersion (Institution of Chemical Engineers, 1999).

[5] S.R. Hanna, P.J. Drivas, \& J.C. Chang, Guidelines for use of vapor cloud dispersion models (New York, NY: Center for Chemical Process Safety/AIChE, 1996). 
[6] O.F.T. Roberts, The theoretical scattering of smoke in a turbulent atmosphere. Proceedings of the Royal Society (London), $104,1923,640-654$.

[7] M.L. Barad, Project prairie grass: A field program in diffusion, Vol. 1, Technical Report AFCRC-RT-58-235, USAF Cambridge Research Laboratory, Bedford, MA, 1958.

[8] M.L. Barad, Project prairie grass: A field program in diffusion, Vol. 2, Technical Report AFCRC-RT-58-235, USAF Cambridge Research Laboratory, Bedford, MA, 1958.

[9] F. Pasquill, The estimation of dispersion of wind-borne material, Meteorology Magazine, 90, 1961, 33-49.

[10] F.A. Gifford, Atmospheric dispersion calculations using the generalized Gaussian plume model, Nuclear Safety, 2(2), 1960, 56-59.

[11] J.L. McElroy \& F. Pooler, The St. Louis dispersion study Vol. 2: Analysis. Technical Report AP-53, US DHEW, Arlington, 1968 .

[12] R.R. Draxler, Determination of atmospheric diffusion parameters, Atmospheric Environment, 10, 1976, 99-105.

[13] H.E. Cramer, Improved techniques for modeling the dispersion of tall stack plume, Proc. 7th Int. Technology Meeting on Air Pollution Modeling and Its Appliction, 1976, 731-780.

[14] J.S. Irwin, Estimating plume dispersion - A comparison of several sigma schemes. Journal of Climate Applied Meteorolology, 22, 1983, 92-114.

[15] A.J. Cimorelli, S.G. Perry, A. Venkatram, J.C. Weil, R.J. Paine, R.F. Lee, \& W.D. Peters, AERMOD - Description of model formulation, Technical Report, USEPA, NC 27711, 1998.

[16] H.A. Panofski \& J.A. Dutton, Atmospheric turbulence: Models and methods for engineering applications (New York, NY: John Wiley \& Sons, 1984).

[17] J.S. Irwin, A theoretical variation of the wind profile powerlaw exponent as a function of surface roughness and stability, Atmospheric Environment, 13, 1979, 191-194.

[18] G.A. Briggs, Diffusion estimations for small emissions, Technical Report ATDL-106, US Atomic Energy Commission, Oak Ridge, TN, 1974.

[19] G.A. Davidson, Simultaneous trajectory and dilution predictions from a simple integral plume model, Atmospheric Environment, 23, 1989, 341-349.

[20] A.R. Van Ulden, Simple estimates for vertical diffusion from sources near the ground, Atmospheric Environment, 12, 1978, 2125-2129.

[21] F. Pasquill \& F.B. Smith, Atmospheric diffusion, Third Edition (Chichester, West Sussex: Ellis Horwood Series in Environmental Science, 1983).

[22] J.S. Touma, W.M. Cox, H. Thistle, \& J.G. Zapert, Performance evaluation of dense gas dispersion models, Journal of Applied Meteorology, 34(3), 1994, 603-615.

\section{Biographies}

Steven S. Beauchemin is an Associate Professor of Computer Science at the University of Western Ontario. Dr. Beauchemin was awarded the Governor General's Gold medal for his Ph.D. work in 1998. The same year, he received an NSERC (Natural Sciences and Engineering Research Council) Post-Doctoral grant which held at the University of Pennsylvania. He received the Young Investigator Award in 2000, and the Research Excellence and Service Award in 2007; both from CIPPRS (the Canadian Image Processing and Pattern Recognition Society). Dr. Beauchemin believes in scientific cross-pollination.

Hussein O. Hamshari is a M.Sc. Graduate in the Department of Computer Science at the University of Western Ontario. He received his Honors B.Sc. in Computer Science in 2005. His research focusses on Computer Vision applications, specifically, automatic facial feature recognition.

Michael A. Bauer is a Full Professor of Computer Science at the University of Western Ontario. He was Chair of the Department from 1991 to 1996 and from 2002 to 2007. He is a Principal Investigator for Shared Hierarchical Academic Research Computing Network (SHARCNET): a multi-university high performance computing grid. From 1996 to 2001, he was the Associate Vice-President of Information Technology at the University of Western Ontario. His Ph.D. in Computer Science is from the University of Toronto. He also holds B.Sc. and M.Sc. degrees in Computer Science. His research interests include distributed computing, particularly the management of distributed applications and systems, network management, software engineering, and high performance computer networks. 